\title{
Spectral obervations of flare stars TZ ARI and ROSS 867
}

\author{
Kostandian G. R. ${ }^{* 1}$, Melikian N. D. ${ }^{\dagger 1}$, Natsvlishvili R. Sh. ${ }^{\ddagger 2}$, and Paronyan G. M. ${ }^{\S 1}$ \\ ${ }^{1}$ V .A. Ambartsumian Byurakan Astrophysical Observatory, Armenia \\ ${ }^{2}$ E. K. Kharadze Abastumani Astrophysical Observatory, Georgia
}

\begin{abstract}
The results of spectral observations of flare stars TZ Ari and Ross 867B are presented. Observations have been carried out by the $2.6 \mathrm{~m}$ telescope of Astrophysical Byurakan Observatory in 1999, 2001 and 2018. The spectral cameras "ByuFOSC" and "SCORPIO" were used during the observations. A strong change of emission lines detected on TZ Ari in 2018, while at the same time the brightness changes of the star in all measured spectral ranges are within the framework of measurement errors. Slight variations of brightness and $E W_{H \alpha}$ are detected in quiescent state of star during the observations in 1999. A strong flare of Ross 867B were observed, with a duration of more than $20 \mathrm{~min}$. In this case if $E W_{H \beta}$ shows strong change, when its maximum value corresponds to the photometric maximum of flare, at the same time $E W_{H \alpha}$ shows decrease, similar to that, observed earlier during the flares of stars EV Lac, HU Del, CM Dra and WX UMa.
\end{abstract}

Keywords: variable star, T Tau stars, UV Ceti stars, spectroscopy,TZ ARI,Ross 867B

\section{Introduction}

The majority of the stars in Galaxy are flare stars. They are on, or very close to the main sequence with spectral types ranging from early $\mathrm{G}$ to late $\mathrm{M}$. The phenomenon of stellar flares was discovered accidentally by Hertzsprung (Hertzsprung, 1924). This discovery was long period forgotten until A. H.Joy and M. L. Humason (Joy \& Humason, 1949) detect similar phenomena on UV Cet, Yz CMi and WX UMa. More than 100 flare stars are known recently in the solar neighborhood, most of which lie within 20pc of the Sun (Pettersen, 1991), and majority are emission line stars. The term "emission line stars" refers to the presence of hydrogen lines in emission in the optical spectrum, indicators of intense chromospheric heating (Cram \& Giampapa, 1987, Cram \& Mullan, 1979).

Recently there has been increased interest in flare stars. New flare stars, as well as new regions of star formation that are rich in UV Ceti-type flare stars and young irregular T Tau-type variables, have been discovered. The simultaneous presence of these two types of young stars in young stellar systems confirms their youth and their possible evolutionary relationship (Ambartsumian, 1954, Ambartsumyan, 1970). Studies of flare stars at the Byurakan Observatory began in the 1960's. When possible, spectral studies were also made of UV Ceti type stars in the neighborhood of the sun and of flare stars in young stellar systems (Melikian, 2014, Melikian et al., 2006, 2011, 2012, 2013, Melikyan et al., 1994, 1995, Tamazian et al., 2005). Since 1999, as a part of various observational programs at the 2.6-m telescope of the Byurakan Observatory, about 140 spectra have been obtained for 9 flare stars in the neighborhood of the sun and in the Orion association. Some data on the flares have been published. These data mostly confirm the results of earlier spectral studies of stellar flares. But there are also some interesting new results.

In this paper the results of spectral studies of two known flare stars in the neighborhood of the sun TZ Ari and Ross 867B are presented.

\footnotetext{
*kgayane@gmail.com

${ }^{\dagger}$ nmelikia@bao.sci.am, Corresponding author

${ }^{\ddagger}$ rezonats@yahoo.com

§paronyan_gurgen@yahoo.com
} 

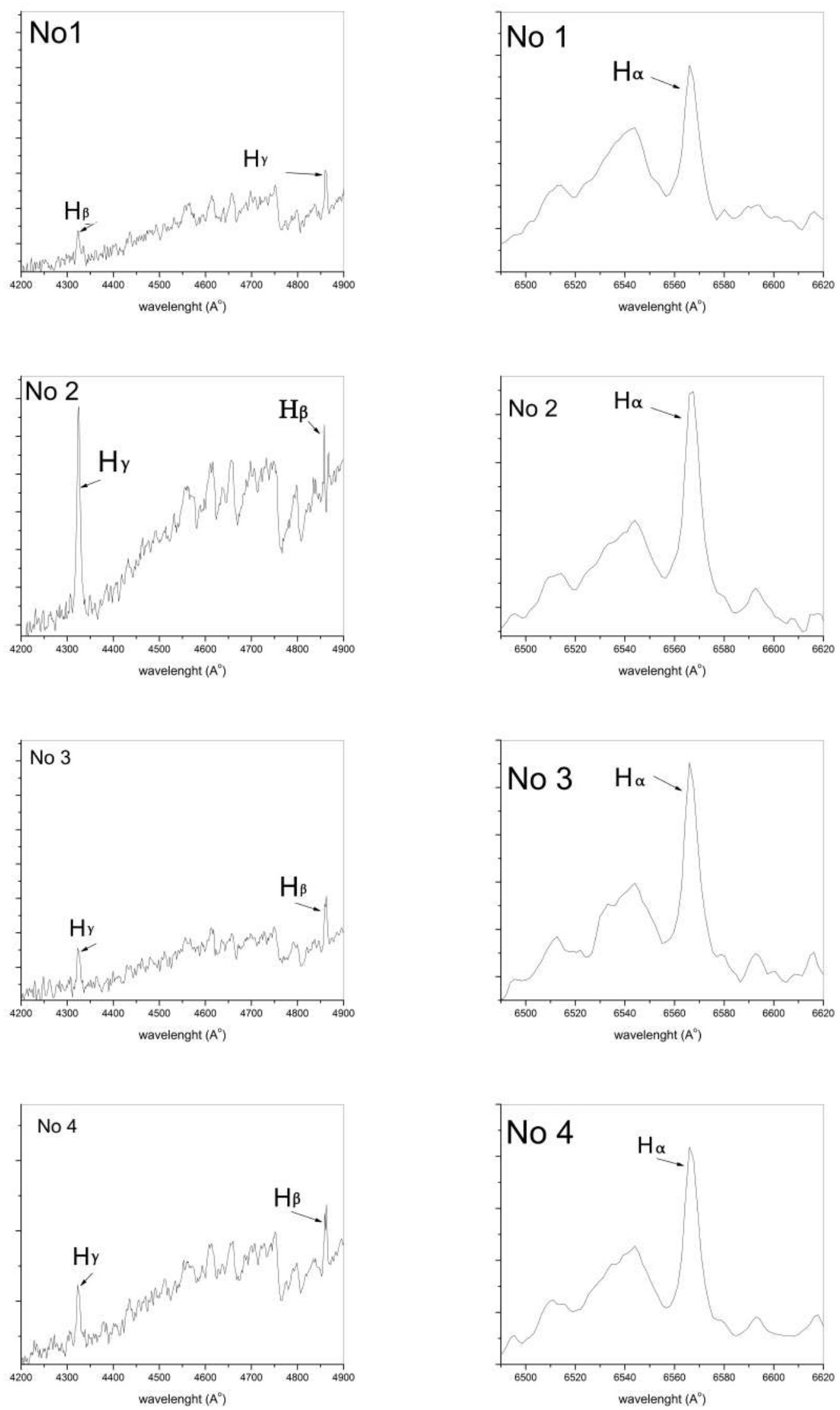

Figure 1. Spectra of TZ Ari in the the spectral ranges $4200-4900 \AA$ and $6490-6620 \AA$ 


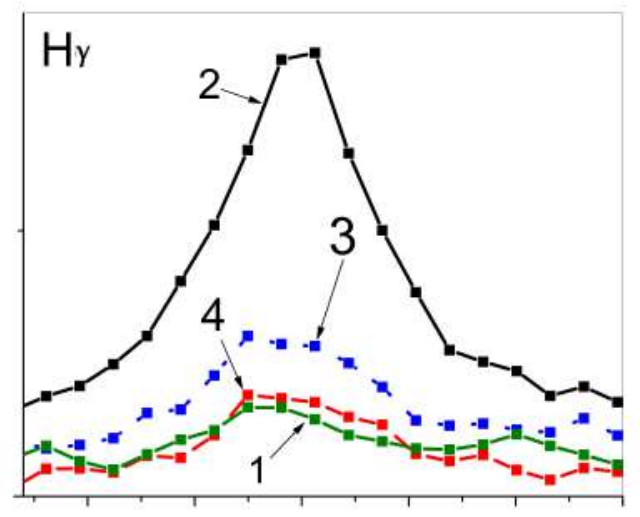

Figure 2. The profiles of emission line $\mathrm{H} \gamma$ on four spectra. The number of spectra are shown by arrows.

\section{Observations}

The spectral observations for the stars TZ Ari and Ross 867 was obtained with the 2.6-m telescope in 1999, 2001 and 2018. 27 spectra were obtained for the program stars during $3^{h} 40^{m}$ effective time of observations. Observations in 1999 were carried out with the "ByuFOSC" detecting system using the 1060x1028 pixel "Thomson" CCD with grism (spectral range $5450-7550 \AA$, dispersion $1.7 \AA /$ pixel) (Movsessian et al., 2000). The observations with the SCORPIO spectral camera were carried out in 2001 and 2018. It employs a $2058 \times 2063$ pixel CCD and covers a spectral range of $4050-7250 \AA$. The net field of the telescope is $14 \mathrm{x} 14$ ' with a spatial resolution of 0.42 " / pixel. The spectra were obtained using a grism with a 600 lines $/ \mathrm{mm}$ grating and an ultimate linear dispersion of $1.7 \AA /$ pixel. The operation of the SCORPIO spectral camera has been described in detail by Afanas'ev et al. (Afanasiev et al., 2005). Preliminary processing of the observational data (background subtraction, radiative flux and wavelength calibrations) was carried out with a program package developed specially for data taken by SCORPIO (Afanasiev et al., 2005, Moiseev, 2002). A neon lamp was used for wavelength calibration during the observations. The quality of the images during the observations was 2 angular seconds or less. A spectrum of the standard star was taken with each series of spectra. The variation in brightness was estimated using the integral emissions of the star and of a standard in the chosen spectral range. It was assumed that the minimum values of the ratios of the recorded integral fluxes of the program star and the standard correspond to the minimum brightness's of the stars. The errors in measuring the amplitudes of the light variation in the star's brightness fluctuate within limits of $\sigma(\Delta \mathrm{m})=0^{m} 1-0^{m} 2$, and the error in measuring the equivalent widths of the emission lines are within $\sigma(\mathrm{EW})=0.3-1.0 \AA$, depending on the exposure time and the brightness of the star.

In Table $1 \log$ of the program stars observations carried out with the $2.6 \mathrm{~m}$ telescope of Byurakan observatory presents. In the columns of the table list are the designations of the stars, observation dates, apparatus used, number of spectra obtained, exposure for each spectrum, and observed spectral range.

Table 1. The log of observations.

\begin{tabular}{|c|c|c|c|c|c|}
\hline Star & Obs.Date & Instrument & $\mathrm{N}$ & Exp.time (min.) & Spect.range \\
\hline TZ Ari & 04.12 .1999 & ByuFOSC & 10 & $5 \mathrm{~m}$ & $5400-7600$ \\
TZ Ari & 05.12 .1999 & ByuFOSC & 10 & $5 \mathrm{~m}$ & $5400-7600$ \\
TZ Ari & 26.11 .2018 & SCORPIO & 4 & $3 \mathrm{~m}$ & $4050-7000$ \\
Ross 867B & 06.09 .2001 & SCORPIO & 3 & $10 \mathrm{~m}$ & $4050-7250$ \\
\hline
\end{tabular}




\section{Observational results}

Over approximately 3.7 hours of total effective time of observational period, 27 spectra for the program stars TZ Ari and Ross 867B were obtained. During the observations a strong changes of emission lines was observed on TZ Ari in 2018 and a strong flare of brightness and strong changes of emission lines detected in case of star Ross 867B in 2001. The detail results of the observations for each star are presented separately below.

\subsection{TZ Ari}

The known flare star TZ Ari (=Gl83.1=L1159-16) is a red dwarf with a large proper-motion (van de Kamp et al., 1966) and with an apparent visual magnitude of $12^{m} 29$. This star known as a flare star (Gershberg, 1970, Pettersen, 1991) which lies relatively close to the Sun at a distance of about 4.4pc. To all probability detected emission in $\mathrm{H} \alpha$ has variable character: it changes in large interval from $E W_{H \alpha}=0 \AA$ (Davison et al., 2015) up to $E W_{H \alpha}=6.33 \AA$ (Rauscher \& Marcy, 2006). Different estimation of spectral type of TZ Ari - from dM3.5 (Fuhrmeister et al., 2018) up to dM8e (Agrawal et al., 1986) were reported as well. These results indicating that changes of spectral characteristics taking place not only during the flare events but also during the quiescent state of the star.

As one can see in table 1 four spectra of TZ Ari have been obtained in 2018. In fig.1 the spectral ranges 4290-4900 $\AA$ (including the emission lines $H \gamma$ and $H \beta$ ) and 6490-6620 $\AA$ (around $H \alpha$ ) from every obtained spectrum are presented. On each spectrum in Fig.1 the number of observed spectrum is presented, as well as hydrogen emission lines in this spectral region are shown by the arrows. On the second spectrum we detect a very strong rise of $H \gamma$ emission line intensity. Unfortunately on the second spectrum, just in the middle of $H \beta$ emission line there is a cosmic defect that we could not rid of during processing the spectrum. As it can be seen not so strong as $H \gamma$, no enough strength increase of $H \alpha$ emission line was detected as well.

In Table 2 the measured values of equivalent widths (EW) of emission lines $H \alpha, H \beta$ and $H \gamma$ on four obtained spectra are presented. In the Table 2 the number of obtained spectra and the start time for every exposition in UT, as well as the measured amplitudes of brightness variation of star in three spectral ranges $(4000-5000 \AA ; 5000-6000 \AA$ and $6000-7000 \AA)$ are given. It must be pointed out that the exposition time for every spectra equal to $180 \mathrm{sec}$. Four spectra were obtained during 18 minutes, typical average duration for flare events of UV Ceti type flare stars. As one can see the brightness changes of the star in all measured spectral ranges are within the limits of measurement errors $\left(0^{m} 1\right.$ $-0^{m} 2$ ), while the emission lines show the strongest changes, similar to stellar flares. Specially strong changes were recorded in equivalent width of the emission line of $H \gamma$, which increased during almost 3 minutes more than 4 times. At the same time, $E W_{H \alpha}$ increases less than twice.

Table 2. EW of measured emission lines.

\begin{tabular}{|c|c|c|c|c|c|c|c|}
\hline Sp & T (start) & $\Delta \mathrm{m}$ & $\Delta \mathrm{m}$ & $\Delta \mathrm{m}$ & $E W_{H \alpha}$ & $E W_{H \beta}$ & $E W_{H \gamma}$ \\
& $\mathrm{UT}$ & $4000-5000 \AA$ & $5000-6000 \AA$ & $6000-7000 \AA$ & & & \\
\hline 1 & $19^{h} 59^{m}$ & $0^{m} 00$ & $0^{m} 04$ & $0^{m} 15$ & $4.0 \AA$ & $3.6 \AA$ & $6.7 \AA$ \\
2 & $20^{h} 05^{m}$ & $0^{m} 22$ & $0^{m} 00$ & $0^{m} 003$ & $7.3 \AA$ & $>5 \AA$ & $28.5 \AA$ \\
3 & $20^{h} 09^{m}$ & $0^{m} 02$ & $0^{m} 04$ & $0^{m} 00$ & $6.4 \AA$ & $5 \AA$ & $18.8 \AA$ \\
4 & $20^{h} 13^{m}$ & $0^{m} 02$ & $0^{m} 00$ & $0^{m} 07$ & $5.6 \AA$ & $4.3 \AA$ & $12.5 \AA$ \\
\hline
\end{tabular}

In Fig. 2 the profiles of the $\mathrm{H} \gamma$ emission line in different observed spectra are presented. In the figure the numbers of the spectra are shown by the arrows. The numbers in the figure correspond to the numbers from Table 2. As can be clearly seen in the figure, during rather short observational time $(\sim 18 \mathrm{~m})$, not only strong changes in the $\mathrm{H} \gamma$ emission line intensity, but the changes of symmetry of its profile, also were detected.

To study the behavior of the $\mathrm{H} \alpha$ emission line in quiescent state of $\mathrm{TZ}$ Ari, we have 20 spectra obtained in 1999 during 2 nights. The spectra were registered in spectral range 5400-7600 $\AA$. It giving possibility to follow for the behavior of emission line $\mathrm{H} \alpha$ only. In Table 3 the results of measurements, the amplitudes of the integral light variation in spectral region $5500-7500 \AA$ in stellar magnitudes 

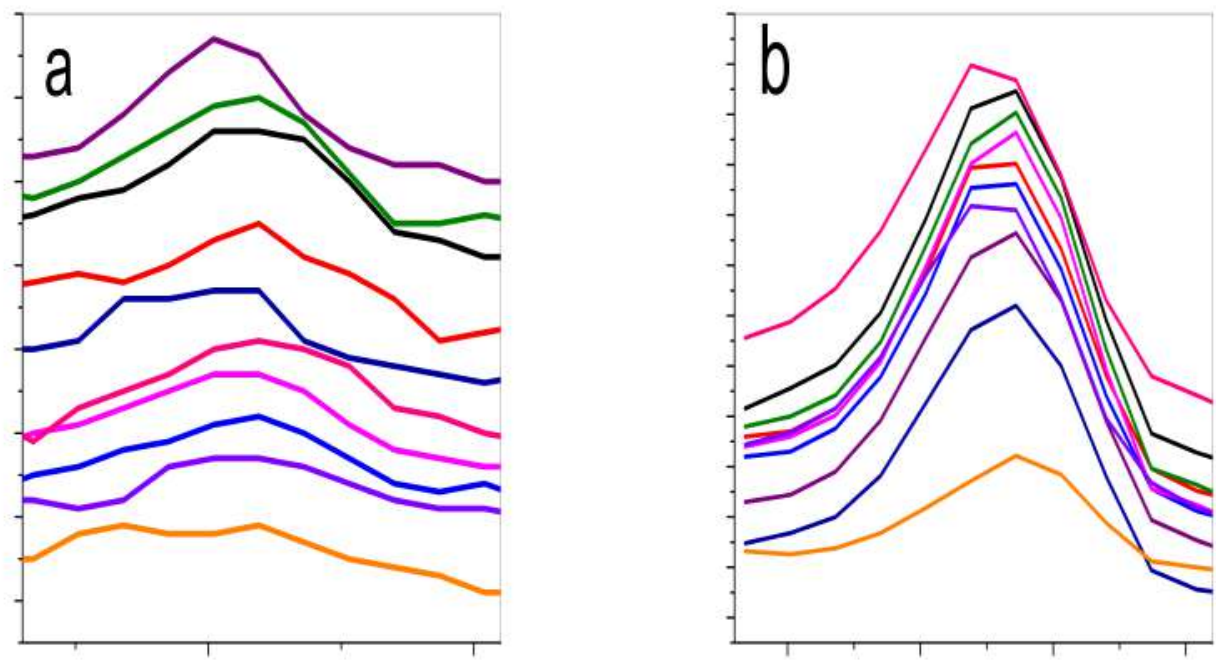

Figure 3. $\mathrm{H} \alpha$ emission line profiles for 2 nights of observations in the same spectral range $6553-6571 \AA$

$(\Delta \mathrm{m})$, and values of $E W_{H \alpha}$ on every spectra in angstroms are presented. In the Table3 the date of observations, number of obtained spectra (No) and start time for every exposition $T_{\text {start }}$ in UT are given as well. As can be seen from the data of Table 3, the measured amplitudes $(\Delta \mathrm{m})$ of the star integral brightness and equivalent widths show slight changes not exceeding the measurement errors $(3 \sigma)$. Average value of Ha equivalent width for the first 10 spectra (NoNo 1-10) is $3.04 \AA$, while for the next 10 spectra average value of $E W_{H \alpha}=4.75 \AA$.

Table 3. Variation of brightness and $E W_{H \alpha}$ for 20 spectra..

\begin{tabular}{|c|c|c|c|c|c|c|c|}
\hline \multicolumn{5}{|c|}{04.12 .2005} & \multicolumn{4}{|c|}{05.12 .1999} \\
\hline No & $T_{\text {start }}$ & $5500-7500 \AA$ & $E W_{H \alpha}$ & No & $T_{\text {start }}$ & $5500-7500 \AA$ & $E W_{H \alpha}$ \\
& $\mathrm{UT}$ & $\Delta \mathrm{m}$ & $\AA$ & & $\mathrm{UT}$ & $\Delta \mathrm{m}$ & $\AA$ \\
\hline 1 & $18^{h} 57^{m}$ & $0^{m} 19$ & 2.6 & 11 & $17^{h} 55^{m}$ & $0^{m} 00$ & 4.9 \\
2 & $19^{h} 08^{m}$ & $0^{m} 00$ & 3.6 & 12 & $18^{h} 03^{m}$ & $0^{m} 26$ & 4.8 \\
3 & $19^{h} 15^{m}$ & $0^{m} 07$ & 3.3 & 13 & $18^{h} 09^{m}$ & $0^{m} 14$ & 5.3 \\
4 & $19^{h} 42^{m}$ & $0^{m} 05$ & 2.9 & 14 & $18^{h} 15^{m}$ & $0^{m} 01$ & 5.0 \\
5 & $19^{h} 49^{m}$ & $0^{m} 16$ & 3.4 & 15 & $18^{h} 21^{m}$ & $0^{m} 17$ & 5.0 \\
6 & $19^{h} 57^{m}$ & $0^{m} 06$ & 3.5 & 16 & $18^{h} 27^{m}$ & $0^{m} 17$ & 4.6 \\
7 & $20^{h} 03^{m}$ & $0^{m} 00$ & 2.6 & 17 & $18^{h} 41^{m}$ & $0^{m} 28$ & 4.8 \\
8 & $20^{h} 10^{m}$ & $0^{m} 02$ & 2.9 & 18 & $18^{h} 47^{m}$ & $0^{m} 15$ & 4.4 \\
9 & $20^{h} 17^{m}$ & $0^{m} 19$ & 2.6 & 19 & $18^{h} 53^{m}$ & $0^{m} 24$ & 4.5 \\
10 & $20^{h} 25^{m}$ & $0^{m} 06$ & 3.0 & 20 & $18^{h} 59^{m}$ & $0^{m} 14$ & 4.2 \\
\hline
\end{tabular}

In Fig.3a,b the profiles of $\mathrm{H} \alpha$ emission line for first (3a) and second (3b) observational nights separately are presented. It must be noted that every spectrum has been received with 5 minutes exposure time. Variation of asymmetry of $\mathrm{H} \alpha$ profiles as well as its intensity changes one can see on the figure.

\subsection{Ross $867 \mathrm{~B}$}

The results obtained from the spectral observations of Ross $867 \mathrm{~B}$ are presented. Ross $867 \mathrm{~B}$ $(=\mathrm{V} 639 \mathrm{Her}=\mathrm{Gl669B})$ is a flare star with a spectral class dM4.5e (Gershberg, 1970, Kunkel, 1967). Ross $867 \mathrm{~B}$ is a member of a wide visual binary system (separation - 22"), with an apparent visual 
magnitude $12^{m} 97$, and lies at a distance of $10.8 \mathrm{pc}$ from the Sun (Pettersen, 1991). At the quiescent state of star a variation of 0.15 magnitudes was observed in the V-band with a period 1.95 days (Doyle et al., 1986). Despite the fact that Ross 867B has been known as a flare star for a long time, it was not studied so often.

3 spectra of Ross $867 \mathrm{~B}$ was observed in 2001 with equal exposure times - 10 minute. Accidently, a brightness increase with the amplitude $\Delta \mathrm{m}(\lambda \lambda 4050-7200 \AA)=1^{m} 7$ is detected during the observations. The continuum and emission lines intensity changes are detected during the flare. In fig.4 the obtained spectra are presented. In figures the number of spectrum is presented, and, hydrogen emission lines are shown by the arrows. The signal/noise ratio do not allowed to measure $\mathrm{EW}$ for $\mathrm{H} \gamma$. A strong change shows emission line $\mathrm{H} \beta$ on the second spectra.
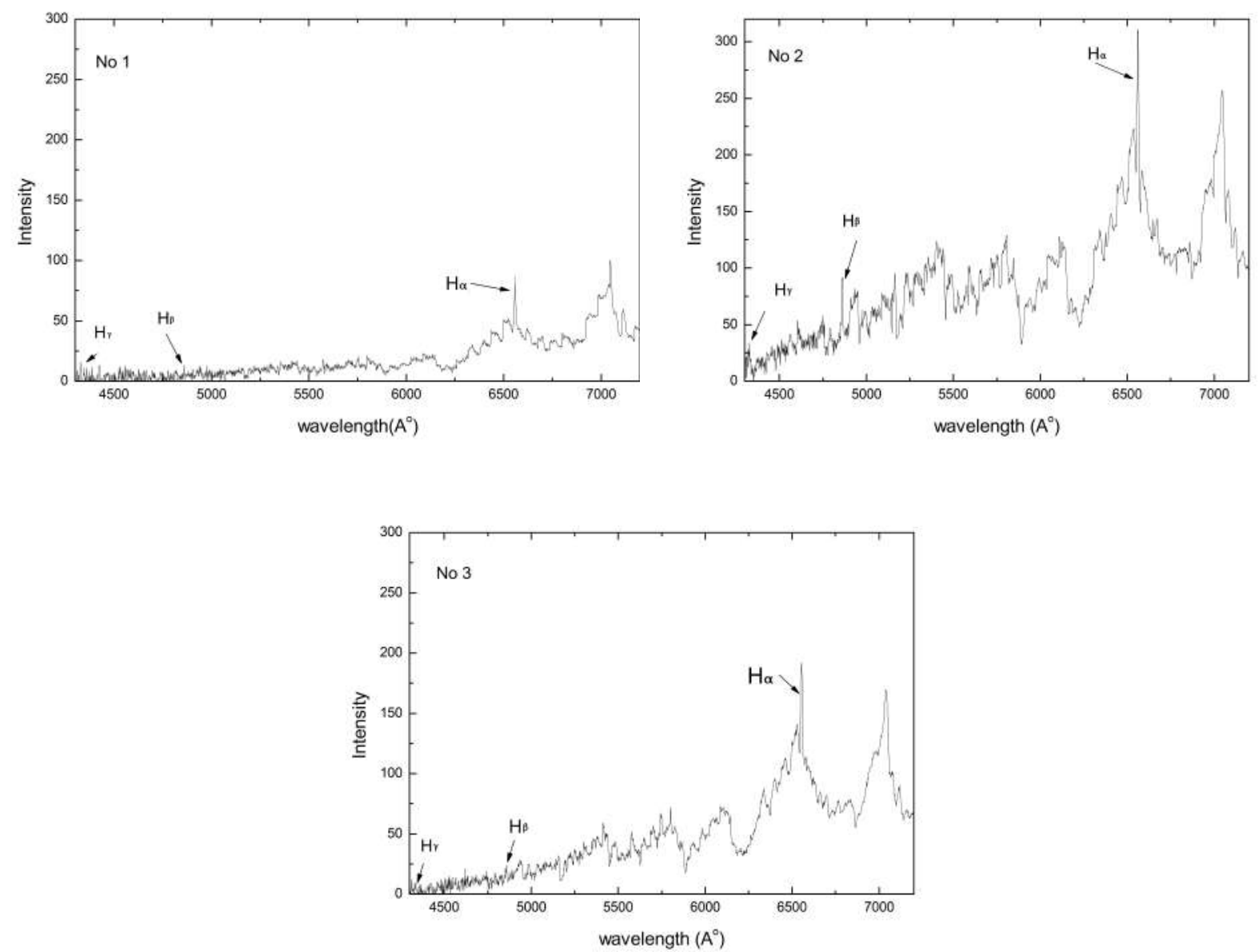

Figure 4. Obtained spectra of Ross 867B

In Table 4 the measured values of equivalent widths (EW) of emission lines $\mathrm{H} \alpha$ and $\mathrm{H} \beta$, as well as amplitudes of brightness variation of star measured on obtained spectra in the spectral ranges $\Delta \mathrm{m}_{1}(\lambda \lambda 4050-5200 \AA), \Delta \mathrm{m}_{2}(\lambda \lambda 5200-6200 \AA)$ and $\Delta \mathrm{m}_{3}(\lambda \lambda 6200-7200 \AA)$ are presented. The errors in measuring of the star's amplitudes is $\sigma_{\Delta m}=0^{m} 1-0^{m} 2$, and the equivalent widths of emission lines are measured with errors $\sigma_{E W}=0.5-0.8 \AA$.

In the Table 4 the number of obtained spectra (Sp.No) and the start time - $\mathrm{T}_{s t}$. for every exposition in UT, as well as the measured amplitudes of brightness variation of star $\left(\Delta \mathrm{m}_{1}, \Delta \mathrm{m}_{2}, \Delta \mathrm{m}_{3}\right)$ in three spectral ranges (4050-5200 $;$; $5200-6200 \AA$ and $6200-7200 \AA$ ) are given. It must be pointed out that the exposition time for every spectra is equal to 10 minute. Four spectra were obtained during 40 minutes. On the first obtained spectrum the star is in quiescent state. As one can see from these data a strong flare were detected with the duration of more than $20 \mathrm{~min}$. The amplitudes of brightness decreases from short to long wavelengths, which is typical for the usual flare events of UV Cet type flare stars. 


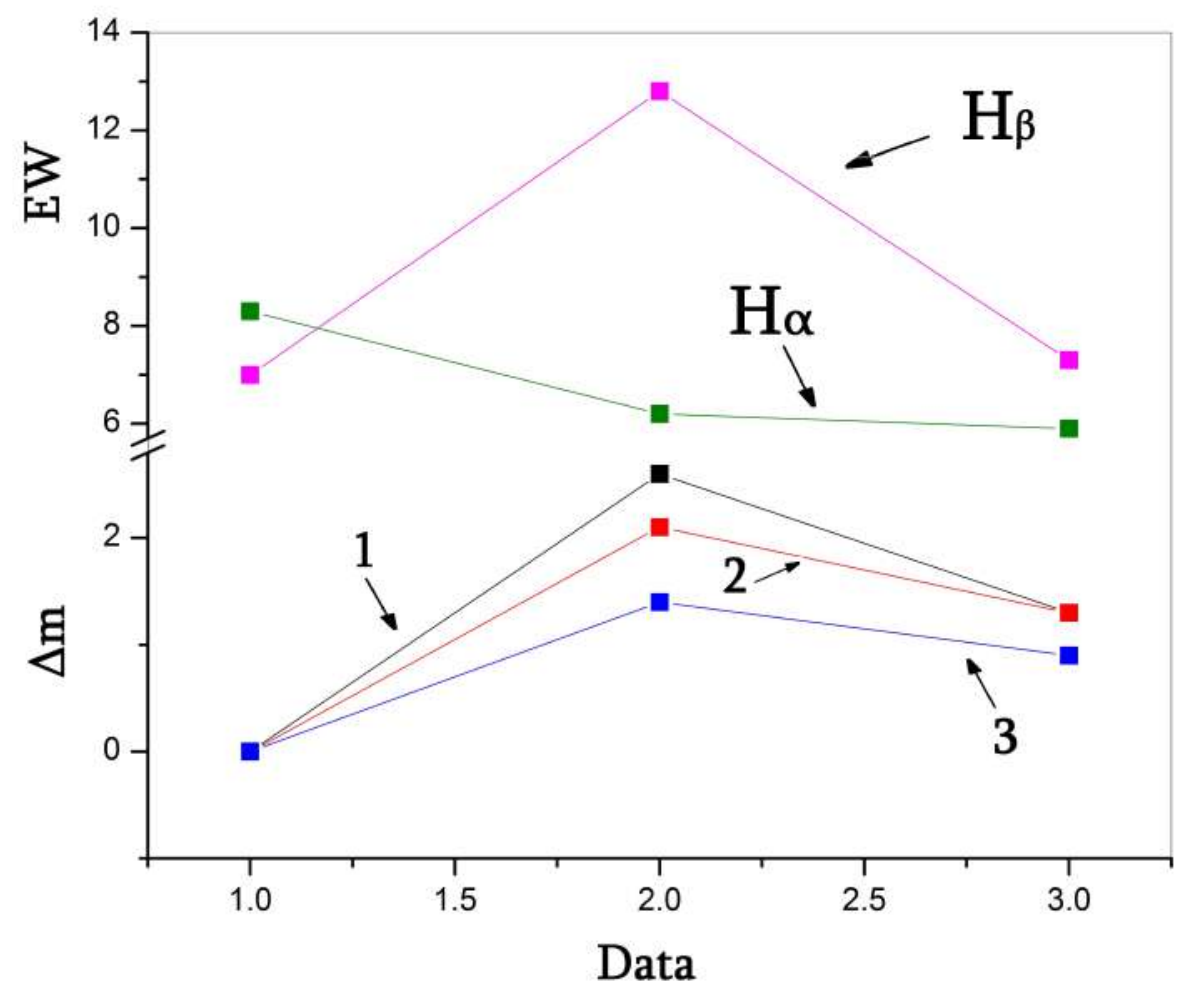

Figure 5. Equivalent widths and brightness variations of Ross 867B during the flare.

The strong changes of equivalent widths also are detected. But if $E W_{H \beta}$ shows flare like variation with the maximum on second spectrum, $E W_{H \alpha}$ shows decrease similar to that observed earlier (Melikian, 2014, Melikian et al., 2006, 2013, Tamazian et al., 2005).

Table 4. Measured amplitudes and equivalent widths of detected flares.

\begin{tabular}{|c|c|c|c|c|c|c|}
\hline $\mathrm{Sp}$ & $\mathrm{T}_{s t}$ & $\Delta \mathrm{m}_{1}$ & $\Delta \mathrm{m}_{2}$ & $\Delta \mathrm{m}_{3}$ & $E W_{H \alpha}$ & $E W_{H \beta}$ \\
$\mathrm{No}$ & $\mathrm{UT}$ & $4050-5200 \AA$ & $5200-6200 \AA$ & $6200-7200 \AA$ & $\AA$ & $\AA$ \\
\hline 1 & $20^{h} 28^{m}$ & $0^{m} 0$ & $0^{m} 0$ & $0^{m} 0$ & 8.3 & 7.0 \\
2 & $20^{h} 45^{m}$ & $2^{m} 6$ & $2^{m} 1$ & $1^{m} 4$ & 6.2 & 12.8 \\
3 & $20^{h} 58^{m}$ & $1^{m} 3$ & $1^{m} 3$ & $0^{m} 9$ & 5.9 & 7.3 \\
\hline
\end{tabular}

The variations in the brightness and equivalent widths are illustrated on Fig.5. On fig.5 equivalent widths are shown by the arrows. Bellow in the figure the brightness variation in different spectral

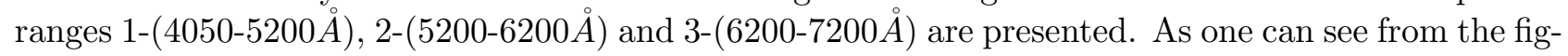
ure maximum value of $E W_{H \beta}$ corresponds to the photometric maximum of flare, while at photometric maximum $E W_{H \alpha}$ decreases.

\section{Conclusion}

During the spectral observations of flare stars TZ Ari and Ross 867B two flares were detected. In case of TZ Ari flare-like strong changes are detected of hydrogen emission lines during the observations carried out in 2018, while at the same time the brightness changes are within the observational errors. During 3 minutes $E W_{H \gamma}$ increases from $6.7 \AA$ (first spectrum) to $28.5 \AA$ (second spectrum), at the same time $E W_{H \alpha}$ show changes from $4 \AA$ to $7.3 \AA$. The duration of flare like variation of these emission lines 
is more than $10 \mathrm{~min}$.

A strong flare is detected on Ross $867 \mathrm{~B}$. The continuum and emission lines intensity changes are detected during the flare. During the flare the measured amplitudes of variation decreases from short to longer wavelengths. But if maximum of $E W_{H \beta}$ variation corresponds to the photometric maximum of flare, the maximum value of $E W_{H \alpha}$ at photometric maximum decreases, similar to that observed earlier on flare stars EV Lac, HU Del, CM Dra and UX UMa (Melikian, 2014, Melikian et al., 2006, 2013, Tamazian et al., 2005).

\section{References}

Afanasiev V. L., Gazhur E. B., Zhelenkov S. L., Moiseev A. V., 2005, Bull. Special Astrophys. Obs., 58,90

Agrawal P. C., Rao A. R., Sreekantan B. V., 1986, MNRAS, 219, 225

Ambartsumian V. A., 1954, Communications of the Byurakan Astrophysical Observatory, 13, 3

Ambartsumyan V. A., 1970, Astrofizika, 6, 31

Cram L. E., Giampapa M. S., 1987, ApJ, 323, 316

Cram L. E., Mullan D. J., 1979, ApJ, 234, 579

Davison C. L., et al., 2015, AJ, 149, 106

Doyle J. G., Byrne P. B., Butler C. J., 1986, Flare activity and by-Draconis type variability on the late-type dMe star gliese 867B. p. 231, doi:10.1007/3-540-16763-3"181

Fuhrmeister B., et al., 2018, A\&A, 615, A14

Gershberg R. E., 1970, Flares of red dwarfs.

Hertzsprung E., 1924, Bulletin of the Astronomical Institutes of the Netherlands, 2, 87

Joy A. H., Humason M. L., 1949, PASP, 61, 133

Kunkel W. E., 1967, PhD thesis, THE UNIVERSITY OF TEXAS AT AUSTIN.

Melikian N. D., 2014, Astrophysics, 57, 77

Melikian N. D., Tamazian V. S., Docobo J. A., Karapetian A. A., Natsvlishvili R. S., 2006, Astrophysics, 49, 488

Melikian N. D., Tamazian V. S., Karapetian A. A., Samsonyan A. L., 2011, Astrophysics, 54, 377

Melikian N. D., Natsvlishvili R. S., Tamazian V. S., Karapetian A. A., 2012, Information Bulletin on Variable Stars, 6031, 1

Melikian N. D., Tamazian V. S., Natsvlishvili R. S., Karapetian A. A., 2013, Astrophysics, 56, 8

Melikyan N. D., Tsvetkov M. K., Sarkisyan R. A., 1994, Astrophysics, 37, 312

Melikyan N. D., Ambaryan V. V., Grankin K. N., Yakobov S. D., 1995, Astrophysics, 38, 103

Moiseev A. V., 2002, Bulletin of the Special Astrophysics Observatory, 54, 74

Movsessian T., Gach J. L., Boulesteix J., Zhamkotsian F., 2000, Baltic Astronomy, 9, 518

Pettersen B. R., 1991, Memorie della Societa Astronomica Italiana, 62, 217

Rauscher E., Marcy G. W., 2006, PASP, 118, 617

Tamazian V. S., Melikian N. D., Karapetian A. A., Natsvlishvili R. S., 2005, Astrophysics, 48, 279

van de Kamp P., Yang C.-Y., Worth M. D., 1966, PASP, 78, 167 